\title{
Clinical Informaticist
}

National Cancer Institute

\section{Source}

National Cancer Institute. Clinical Informaticist. NCI Thesaurus. Code C154708.

An individual that designs, implements, evaluates and/or analyzes information technology

in a healthcare or research setting. 